\title{
How can novel microscopic approaches shed light on the function of nucleic acid-based drugs?
}

\author{
"State-of-the-art approaches leverage fluorescent proteins across the visible \\ spectrum, surpass the resolution limits of visible light and achieve sub-Ångström \\ resolution with electron microscopy."
}

\begin{abstract}
Keywords: confocal $\bullet$ Cryo-EM • EDS $\bullet$ FRET $\bullet$ RNAi $\bullet$ siRNA $\bullet$ super-resolution $\bullet$ TEM - TIRF
\end{abstract}

The focus on the potential of nucleic acidbased therapeutics has grown rapidly since the initial characterization of RNAi [1]. Recent excitement about adapting CRISPR genome editing for therapeutic purposes has furthered that growth and interest [2]. Parallel to the development of these nucleic acid-based drugs has been the development of increasingly sensitive approaches for the analysis of their function. Among these new approaches are advanced and novel applications of microscopy [3]. Stateof-the-art approaches leverage fluorescent proteins across the visible spectrum, surpass the resolution limits of visible light and achieve sub-Ångström resolution with electron microscopy. Application of these tools to the analysis of the function of nucleic acid-based drugs has provided new insights and perspectives that will guide their future development and clinical application.

\section{Transmission electron microscopy}

In biological systems, transmission electron microscopy (TEM) is often used to determine the intracellular locations of delivered materials with achievable resolution down to $0.2 \mathrm{~nm}$. Because of the low electron density of the majority of biological molecules, samples are typically stained with electron dense elements to enhance image contrast, with immunogold labeling often being used to visualize target molecules. Recent advances in the tracking of gold labels has been utilized to monitor the intracellular trafficking of nucleic acids and better understand the roles of endogenous and exogenous nucleic acids in biological processes.

Historically, immunogold labeling was achieved through a combination of primary and secondary antibodies [4], resulting in a loss in resolution in assigning the location of the target molecule. Recent improvements in the use of primary antibodies alone and in direct labeling of nucleic acids with gold nanoparticles has resulted in improved TEM resolution. In one case, researchers were studying a correlation between Alzheimer's disease (AD) and the snRNAs of spliceosomes [5]. Using TEM and immunogold labeling of the snRNAs, they showed that snRNAs form aggregates associated with the AD marker protein tau and that snRNA aggregation was specific to $\mathrm{AD}$ (i.e., not found in samples from other neurodegenerative diseases). Gold-labeled siRNAs were used to assess the intracellular trafficking of lipid nanoparticles in siRNA delivery [6]. The investigators visualized the intracellular location of the siRNAs at multiple time points. Leveraging the resolution of TEM, the investigators could track the siRNAs across multiple organelles, quantify the delivery efficiency of the lipid nanoparticles, calculate the kinetic parameters associated with siRNA release and identify the rate-limiting step in siRNA delivery. Early attempts to visualize biological molecules within nuclei revealed that immunogold labels were unable to pass the nuclear envelope. Conjugating monoclonal antibodies to gold particles of various sizes, it was found that nuclear transport could be achieved using 0.8 -nm gold
Daniel Vocelle

Department of Chemical Engineering \& Materials Science, Michigan State University, 428 S. Shaw Lane, Room 2527, East Lansing, MI 48824, USA

\section{Christina Chan}

Department of Chemical Engineering \& Materials Science, Michigan State University, 428 S. Shaw Lane, Room 2527, East Lansing, MI 48824, USA

and

Departments of Biochemistry \& Molecular Biology, Michigan State University, East Lansing, MI 48824, USA

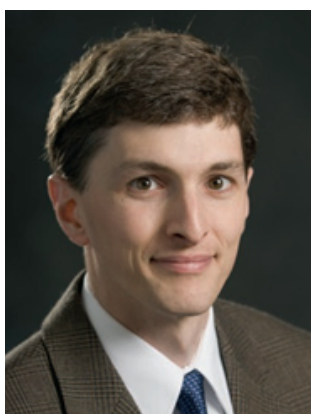

S Patrick Walton

Author for correspondence: Department of Chemical Engineering \& Materials Science, Michigan State University, 428 S. Shaw Lane, Room 2527, East Lansing, MI 48824, USA

Tel.: +1 5173555135

Fax: +1 5174321105

spwalton@egr.msu.edu

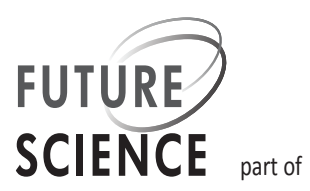


particles [7], indicating that prior efforts were limited by the size of the gold labels. This discovery should greatly enable further applications of TEM to the analysis of nucleic acid delivery, including to nuclei for gene therapy/transfer applications.

Cryo-EM extends the imaging capacity of TEM by preserving a sample's hydrated, near-native structure and reducing damage caused by irradiation. In doing so, cryo-EM provides near atomic resolution (4.5 $\AA$ ). Consequently, single-particle analyses, crystallography and tomography are more common with cryo-EM. In examining siRNA delivery vehicles, cryo-EM was used in conjunction with novel computational analyses to obtain quantitative morphological data on individual lipid nanoparticles [8]. A novel approach, Zernike phase contrast cryo-EM was applied to generate improved structural information on the interactions of Dicer with substrate RNAs, showing that the structure of Dicer changes based on the incorporation of the substrate and interactions of the substrate with other RNA-binding proteins [9].

\section{"Leveraging the resolution of transmission electron microscopy, the investigators could track the siRNAs across multiple organelles, quantify the delivery efficiency of the lipid nanoparticles, calculate the kinetic parameters associated with siRNA release and identify the rate-limiting step in siRNA delivery."}

In combination with TEM, energy dispersive spectroscopy (EDS) provides elemental analysis or chemical characterization of samples. EDS, in this case combined with cryo-EM, was used to identify the intracellular distribution of $\mathrm{pH}$-responsive nanogels loaded with iron oxide nanoparticles and siRNAs [10]. Using EDS to determine the elemental distribution within a TEM image allowed the amount of silica nanoparticles within subcellular compartments to be quantified and compared across four different plant species [11]. A novel method for protein identification and quantification based on a protein's unique ratio of sulfur to other metals has also been developed based on EDS [12]. With this technique, the stoichiometry and structure of a protein-ssDNA complex was determined. With EDS elemental mapping, it is possible to characterize the intracellular events associated with the uptake and trafficking of nucleic acid-based drugs and to identify their targets without the use of immunolabeling.

\section{Fluorescence microscopy}

The evolution of microscopic techniques has also resulted in improved fluorescence-based methods, including confocal microscopy, total internal reflection fluorescence (TIRF) microscopy and fluorescence resonance energy transfer (FRET). Novel instrumentation in combination with advanced computational analyses has been able to push the resolution of confocal microscopy beyond the diffraction limit of visible light, referred to as super-resolution. Super-resolution techniques are subcategorized by refinement of the point-spread function of the recovered signal ( $4 \pi$ and structured illumination microscopy [SIM]), specially patterned excitation (stimulated emission depletion [STED] and saturated structured illumination microscopy [SSIM]), or use of stochastic detection to achieve single molecule fluorescence (stochastic optical reconstruction microscopy [STORM], photoactivated localization microscopy [PALM], and fluorescence photoactivation localization microscopy [FPALM]). Recent efforts toward cost-effective and accessible super-resolution microscopy led to the development of DNA-PAINT (short, stochastic fluorescently-labeled oligonucleotides), which allows sub-10-nm resolution analysis of target DNA sequences [13]. Airyscanning replaces the pinhole aperture in confocal systems with an array of detectors and narrows the point spread function through pixel reassignment. Fully implemented, this technique enhances spatial resolution by a factor of 1.7 over standard confocal microscopy while simultaneously lowering the detection limit of the technique [14].

TIRF microscopy uses an evanescent wave to achieve detection of single molecule fluorescence near the specimen-stage interface. In examining the intracellular events associated with siRNA delivery, TIRF was used in conjunction with multiple particle tracking to identify a number of cellular signals involved in the macropinocytosis of siRNA-containing lipid nanoparticles. In this way, it was found that endocytotic recycling is responsible for the exocytosis of up to $70 \%$ of the delivered siRNAs [15]. Using the single molecule detection and photobleaching capability of TIRF, a novel technique was developed to determine the stoichiometric ratio of molecules within biological complexes. Applying this technique to protein/RNA interactions allowed their stoichiometric ratio and mechanism of interaction to be determined [16].

In combination with imaging techniques, FRET is used to measure the distance between fluorophores or detect the interactions of biological molecules. Recent improvements to fluorophore lifetime and quantum yield have expanded the application of FRET in live cell confocal microscopy. In order to determine the kinetic parameters of intracellular siRNA dissociation, siRNA strands were labeled with FRET pairs. From this assay, it was possible to determine the 
kinetic parameters associated with siRNA diffusion, dissociation and degradation [17]. Using FRET-labeled siRNA as a method for assaying siRNA integrity, it was shown that the cytoplasmic delivery of siRNA is accomplished by burst release from endosomal vesicles [18].

\section{Conclusion}

The future of monitoring the function of nucleic acidbased drugs lies in a combination of increasingly sensitive analytical approaches and novel techniques that will offer high-resolution imaging with high-throughput capacity. Advances in the resolution of electron microscopy will be achieved through enhancing contrast, either through the use of electron-specific detectors or better implementation of electron phase contrast. Further development of computational models, specifically in relation to object identification and $3 \mathrm{D}$ reconstruction, will advance the high-throughput

\section{References}

1 Fire A, Xu S, Montgomery MK, Kostas SA, Driver SE, Mello CC. Potent and specific genetic interference by doublestranded RNA in Caenorhabditis elegans. Nature 391(6669), 806-811 (1998).

2 Pennisi E. The CRISPR Craze. Science 341(6148), 833-836 (2013).

3 Portis AM, Carballo G, Baker GL, Chan C, Walton SP. Confocal microscopy for the analysis of siRNA delivery by polymeric nanoparticles. Microsc. Res. Tech. 73(9), 878-885 (2010).

4 Hermann R, Walther P, Müller M. Immunogold labeling in scanning electron microscopy. Histochem. Cell Biol. 106(1), 31-39 (1996).

5 Hales CM, Dammer EB, Diner I et al. Aggregates of small nuclear ribonucleic acids (snRNAs) in Alzheimer's disease. Brain Pathol. 24(4), 344-351 (2014).

6 Gilleron J, Querbes W, Zeigerer A et al. Image-based analysis of lipid nanoparticle-mediated siRNA delivery, intracellular trafficking and endosomal escape. Nat. Biotechnol. 31(7), 638-646 (2013).

7 Orlov I, Schertel A, Zuber G et al. Live cell immunogold labelling of RNA polymerase II. Sci. Rep. 5, 8324 (2015).

8 Crawford R, Dogdas B, Keough E et al. Analysis of lipid nanoparticles by Cryo-EM for characterizing siRNA delivery vehicles. Int. J. Pharm. 403(1-2), 237-244 (2011).

9 Taylor DW, Ma E, Shigematsu H et al. Substrate-specific structural rearrangements of human Dicer. Nat. Struct. Mol. Biol. 20(6), 662-670 (2013).

10 Marotta R, Falqui A, Curcio A, Quarta A, Pellegrino T. Immunocytochemistry, electron tomography, and energy dispersive X-ray spectroscopy (EDXS) on cryosections of capacity of data analysis from electron microscopy. In light microscopy, the future likely lies in combining techniques, to enhance the throughput of techniques such as TIRF and confocal through the incorporation of plate readers and to improve the resolution and sensitivity of techniques such as imaging flow cytometry through implementation of super-resolution methods like Airyscanning.

\section{Financial \& competing interests disclosure}

This work was supported by the NIH (USA; GM089866 and CA176854) and the National Science Foundation (USA; CBET 0941055, 1510895). The authors have no other relevant affiliations or financial involvement with any organization or entity with a financial interest in or financial conflict with the subject matter or materials discussed in the manuscript apart from those disclosed.

No writing assistance was utilized in the production of this manuscript.

human cancer cells doped with stimuli responsive polymeric nanogels loaded with iron oxide nanoparticles. Methods Mol. Biol. 1025, 179-198 (2013).

11 Sun D, Hussain H, Yi Z et al. Uptake and cellular distribution, in four plant species, of fluorescently labeled mesoporous silica nanoparticles. Plant Cell Rep. 33(8), 1389-1402 (2014).

12 Garman EF, Zeldin OB. Elemental analysis of proteins by proton induced $\mathrm{x}$-ray emission (microPIXE). In: Advancing Methods for Biomolecular Crystallography. Springer Netherlands, 79-89 (2013).

13 Jungmann R, Avendaño MS, Woehrstein JB, Dai M, Shih WM, Yin P. Multiplexed 3D cellular super-resolution imaging with DNA-PAINT and Exchange-PAINT. Nat. Methods. 11(3), 313-318 (2014).

14 Weisshart K. The Basic Principle of Airyscanning. http://confocal-club.ru/upload/article/EN_wp_LSM-880_ Basic-Principle-Airyscan.pdf

15 Sahay G, Querbes W, Alabi C et al. Efficiency of siRNA delivery by lipid nanoparticles is limited by endocytic recycling. Nat. Biotechnol. 31(7), 653-658 (2013).

16 Zhang H, Guo P. Single molecule photobleaching (SMPB) technology for counting of RNA, DNA, protein and other molecules in nanoparticles and biological complexes by TIRF instrumentation. Methods 67(2), 169-176 (2014).

17 Shin S, Kwon H-M, Yoon K-S, Kim D-E, Hah SS. FRET-based probing to gain direct information on siRNA sustainability in live cells: asymmetric degradation of siRNA strands. Mol. Biosyst. 7(7), 2110-2113 (2011).

18 Hirsch M, Helm M. Live cell imaging of duplex siRNA intracellular trafficking. Nucleic Acids Res. 43(9), 4650-4660 (2015). 